\title{
Cysticercosis of the central nervous system: clinical and therapeutic considerations
}

\author{
GONZALO TORREALBA, * SERGIO DEL VILLAR, ${ }^{*}$ PATRICIO TAGLE, ${ }^{*}$ \\ PAULINA ARRIAGADA, $\dagger$ CARLOS S KASE $\ddagger$
}

From the Departments of Neurosurgery* (Drs. Torrealba, Del Villar and Tagle) and Neurology, †Catholic University of Chile, Santiago, Chile, and Department of Neurology, University of South Alabama, College of Medicine, Mobile, Alabama, USA $\ddagger$

SUMMARY In a group of forty cases of cysticercosis of the central nervous system, $59 \%$ presented with intracranial hypertension due to obstructive hydrocephalus. Ventricular or cisternal cysts, and chronic cysticercus meningitis were the most common causes of hydrocephalus. Seizures occurred in $40 \%$ of the patients, in one-half of them in association with CT-detected parenchymatous cysts. In $20 \%$ of the cases progressive mental deterioration was the main clinical feature, at times associated with hydrocephalus. CT scan provided the highest diagnostic yield, being abnormal in $90 \%$ of cases. Long term prognosis was poor, with a mortality rate of $38 \%$ over a 40-month follow-up period. The most common cause of death (60\%) was meningitis. CSF shunting is the treatment of choice for hydrocephalus, irrespective of its mechanism. Surgical resection is indicated in some cases with a single superficial (cortical) or posterior fossa cyst. Supratentorial cysts carry a relatively benign prognosis.

The aetiology of neurocysticercosis, the biological cycle of the parasite, and the pathological lesions it provokes have been well documented. ${ }^{1-10}$ However, some aspects of the disease, such as its initial clinical manifestations, the yield of various diagnostic methods, and its treatment still remain unclear. In addition, accurate figures on outcome and long-term prognosis are not widely available. We report a group of 40 patients with cysticercosis of the central nervous system, in an attempt to clarify these questions.

Forty patients with a diagnosis of neurocysticercosis were studied. Aids to diagnosis included complement fixation reaction in blood and cerebrospinal fluid (CSF), counterimmunoelectrophoresis, and intradermal reactions. These tests were positive in 32 cases. The diagnosis was verified pathologically in 18 cases, and the cause of death was verified by necropsy in 10 cases. Follow-up covered periods from 7 to 97 months (mean: 40 months) in 32 patients.

Received 8 December 1983.

Accepted 10 January 1984

Address for reprint requests: Carlos S Kase, MD Department of Neurology, University of South Alabama, 2451 Fillingim Street, Mobile, Alabama 36617, USA.

\section{Results}

The ages of patients ranged from 9 to 69 years, and 24 patients were under 40 years: The time between onset of symptoms and diagnosis was variable: less than 1 month in 11 cases, less than 6 months in 26 , and less than one year in 30 patients.

The clinical presentation at onset is shown in table 1. The principal signs were (a) Intracranial hypertension (20 patients). In 12 patients it was the only manifestation, and in eight it was associated with mental deterioration or seizures. Thirteen patients developed symptoms of gradually increasing intracranial hypertension over periods shorter than 6 months, whereas six others had an intermittent course with symptoms lasting weeks to months. One other patient had symptoms for 10 years, and funduscopic examination on admission revealed bilateral optic atrophy. In 11 patients papilloedema was detected on initial examination. (b) Seizures (16 patients). Of 11 cases with seizures as the only symptom, eight had generalised seizures, and the remaining three had partial motor seizures. In the remaining five patients seizures were associated with intracranial hypertension, mental deterioration, or motor disorders of acute onset. One patient in this group presented initially as status epilepticus. 
Table 1 Initial clinical syndrome in 40 cases of CNS cysticercosis

\begin{tabular}{lll}
\hline & No. Cases & $\%$ \\
\hline Intracranial hypertension & 20 & 50 \\
Seizures & 16 & 40 \\
Mental deterioration & 9 & 22 \\
Acute motor disorders & 5 & 12 \\
\hline
\end{tabular}

(c) Mental deterioration (nine patients). In six cases the dementia was associated with one of the other forms of presentation. This was generally characterised by a progressive dementia, which in three patients was associated with behavioral abnormalities (intermittent agitation and visual hallucinations). (d) Motor deficits (five patients). These corresponded to hemiplegia or hemiparesis of sudden onset, often not accompanied by sensory changes. A progressive worsening followed the sudden onset in two patients. Aphasia was present in two others, one of whom also presented with seizures. A slow motor improvement over a period of weeks occurred in three patients in this group.

Laboratory investigations are summarized in table 2. Eosinophilia in peripheral blood was an occasional finding. Over half of the patients had abnormal CSF. Eighty percent of patients with CSF pleocytosis had less than 100 cells $/ \mathrm{mm}^{3}$. Polymorphonuclear cells predominated in most cases. The CSF protein elevation rarely exceeded $1 \mathrm{~g} / \mathrm{l}$, but in one case it reached $6.38 \mathrm{~g} / 1$. In only three cases was there an albumin/cell dissociation.

Seventeen of 25 angiograms performed were abnormal. Fourteen of them showed hydrocephalus, and expanding mass lesions were found in three cases (fig 1). CT scans were performed in 20 cases and the patterns of abnormality varied according to the anatomical character of the lesions (table 3). CT abnormalities (fig 2) were present in $90 \%$ of the cases. In one instance, initial examination showed no abnormality, but on repeat scan one month later multiple small cysts were detected.

The following correlations between clinical syndromes and anatomo-radiological lesions were observed: (a) Intracranial hypertension. The cause of this syndrome was hydrocephalus in 17 patients,

Table 2 Laboratory examinations

\begin{tabular}{lcc}
\hline Examination & No. Cases & $\%$ \\
\hline Blood eosinophilia & $9(38)^{*}$ & 23 \\
CSF & $17(38)$ & 53 \\
Pleocytosis (>5 cells/cu mm) & $15(38)$ & 46 \\
Elevated protein (>30 mg\%) & $17(25)$ & 68 \\
Angiographic changes & $17(19)$ & 90 \\
CT scan abnormalities & 17 \\
\hline
\end{tabular}

*Number in parentheses indicates number of patients who underwent testing.
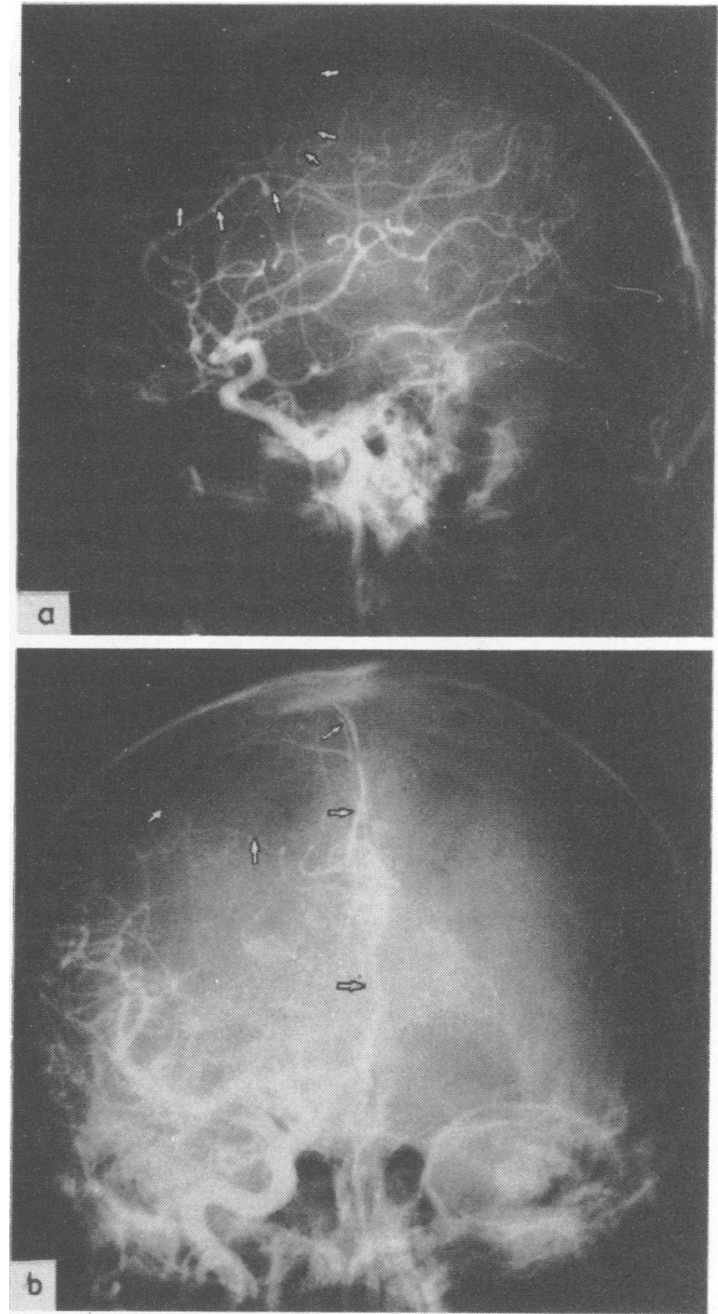

Fig 1 (A) Lateral angiogram, arterial phase, with large avascular area (arrows) corresponding to a large fronto-parietal cysticercus. (B) AP view of same angiogram showing mass effect with midline shift (arrows), due to the large fronto-parietal cyst.

in seven of whom ventricular or cisternal cysts were identified. The remaining 10 patients showed CSF changes consistent with chronic meningitis, the

Table 3 Computed tomography in 20 cases of CNS cysticercosis

\begin{tabular}{lll}
\hline Findings & No. Cases & $\%$ \\
\hline Solitary or multiple cysts & 7 & $35 \%$ \\
Calcifications and cystic images & 4 & $20 \%$ \\
Hydrocephalus and cystic images & 3 & $15 \%$ \\
Pure hydrocephalus & 3 & $15 \%$ \\
Normal & 3 & $15 \%$ \\
\hline
\end{tabular}



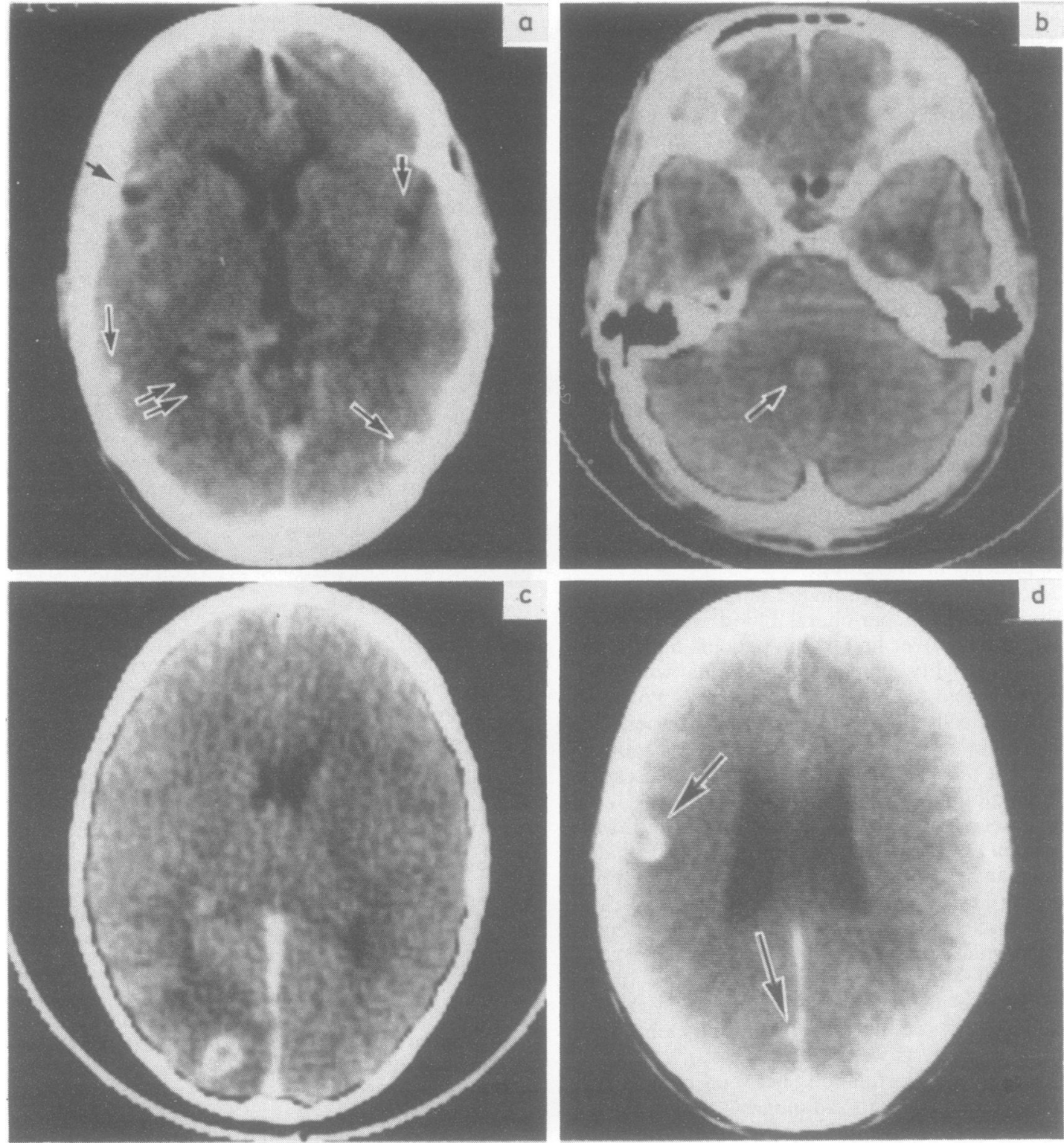

Fig 2 (a) Multiple hypodense (arrowheads) and hyperdense (arrows) lesions scattered in cortical and subcortical areas, in addition to deep seated cysts (double arrows). (b) Intraventricular calcified cyst (arrow) of the fourth ventricle. (c) Solitary subcortical left occipital cyst surrounded by white matter oedema, and showing post-contrast ring enhancement. (d) Subcortical calcified cyst in the left fronto-parietal and occipital areas (arrows) and associated hydrocephalus.

likely cause of the hydrocephalus. In only one case was intracranial hypertension due to multiple large hemispheric cysts. CT scan was not available in two cases with increased intracranial pressure of unknown mechanism. (b) Seizures. Half of the patients with seizures had solitary or multiple parenchymatous cysts. Hydrocephalus was present in four patients in whom seizures were associated with either intracranial hypertension or mental deterioration. (c) Mental deterioration. Hydrocephalus was 


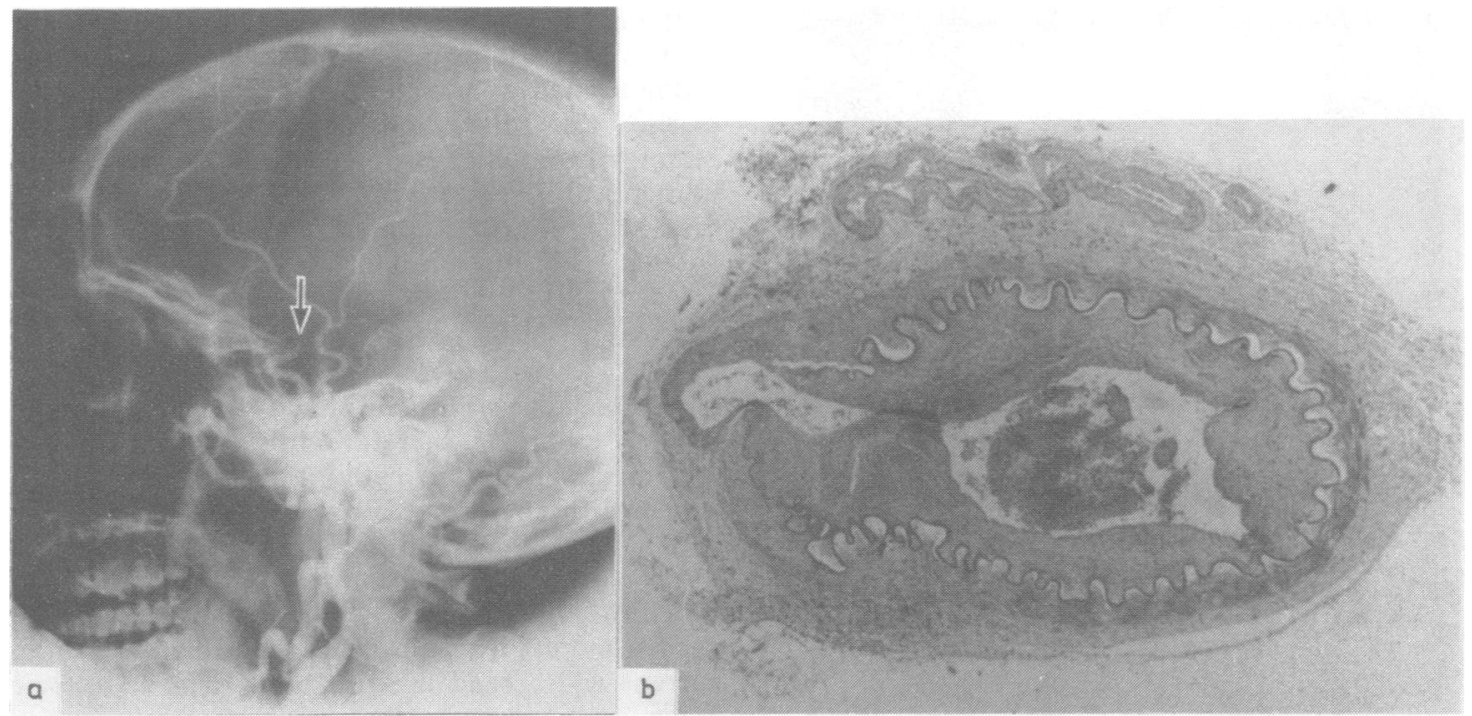

Fig 3 (A) Left lateral carotid angiogram showing occlusion of the supraclinoid internal carotid artery (arrow), caused by endarteritis. (B) Medium-size arterial branch of the circle of Willis with chronic adventitial inflammation and intimal proliferation ("endarteritis obliterans"). ( $H \& E, \times 32)$

present in all nine patients, six of whom had a CSF suggesting basilar cysticercosic meningitis. (d) Acute motor disorders. Different types of lesions accounted for these motor syndromes. A 35-year-old male had a left supraclinoid carotid artery occlusion (fig $3 \mathrm{~A}$ ), and presented with right hemiplegia and aphasia. He underwent at extracranial-intracranial bypass operation and shunt placement for concomitant hydrocephalus, but he deteriorated progressively from chronic meningitis and died 6 months later. Necropsy confirmed meningitis and showed a proliferative form of endarteritis associated with marked stenosis in all proximal branches of the circle of Willis (fig 3B), with multiple left hemispheric cerebral infarcts distributed in the centrum semi-ovale and adjacent cortex. Another patient, a 36-year-old. male, suddenly developed a left hemiparesis that progressed to hemiplegia in 4 days. CT scan revealed a large right fronto-parietal cyst with marked oedema of the adjacent parenchyma (fig 4). In three patients with motor deficits only angiog-

Table 4 Causes of death in 12 cases of CNS cysticercosis

\begin{tabular}{ll}
\hline Cause of Death & No. Cases \\
\hline Basilar meningitis & $7^{*}$ \\
Giant cysticercus & 1 \\
Shunt obstruction & 1 \\
Status epilepticus & 1 \\
Sepsis & 1 \\
Malignant melanoma & 1 \\
\hline
\end{tabular}

*Two cases not verified by necropsy raphic studies were performed, which were normal in two and showed hydrocephalus in the third.

In a group of 32 patients followed for periods of 7 to 97 months (mean 40 months), 12 (38\%) had died, three $(9 \%)$ were severely disabled, and 17 $(53 \%)$ had a benign course. Eight of the 12 deaths were caused by progression of the disease (table 4). The most common cause of death was cysticercosis meningitis. The unfavourable prognosis of this condition was suggested by the correlations of the clinical course with the CSF findings: CSF pleocytosis was present in 3 of 17 cases with benign course, and in 10 of 15 patients who died or had severe sequelae.

\section{Treatment}

Craniotomy was performed in the following cases: (a) Mass lesion of unknown aetiology in the posterior fossa (two cases). On exploration, a cyst was found in the cisterna magna or the 4 th ventricle. No attempt was made to remove the leptomeningeal adhesions after diagnosis, and a CSF shunting procedure was performed. (b) Opto-chiasmatic arachnoiditis with severe and progressive visual loss (two cases). The optic nerves were debrided, and since hydrocephalus was present, a CSF shunt was also performed. (c) Supratentorial mass lesions (seven cases). Cyst resection was performed and in one case CSF shunting was required in addition.

In 16 patients with known hydrocephalus, CSF 

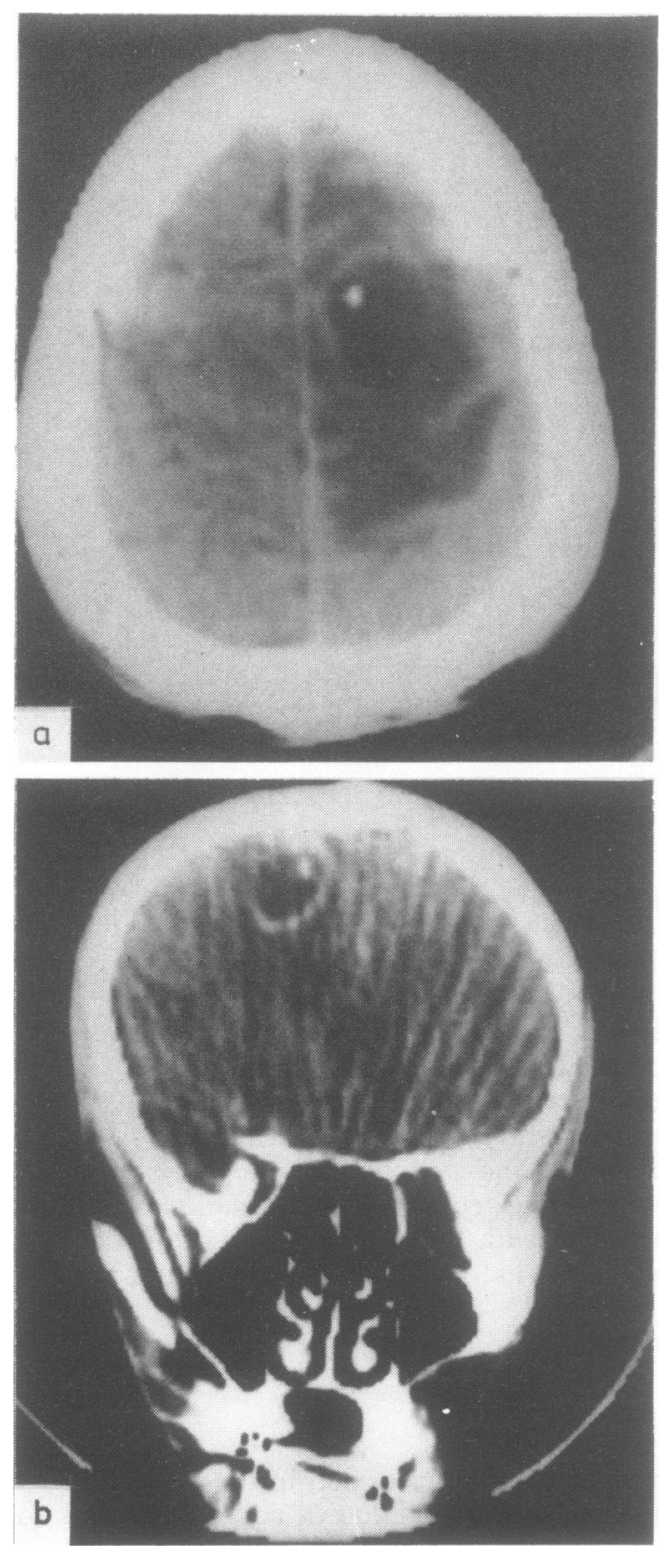

Fig 4 Horizontal $(A)$ and coronal $(B) C T$ views of large right fronto-parietal cyst containing a visible scolex and surrounded by white matter oedema. shunting alone was performed. Only two patients with hydrocephalus were not subjected to shunting owing to their poor clinical condition. Table 5 summarises the outcome of surgically treated cases. Steroids were administered to all patients with intracranial hypertension. The small number of cases treated and the variable doses used preclude an analysis of the effectiveness of steroids.

\section{Discussion}

Neurocysticercosis usually affects the young adult in full active life. ${ }^{9-14}$ Therefore, it is especially important to understand its pathophysiology, and devise proper forms of therapy. In our series, the diagnosis was made within 6 months of the onset of symptoms in $70 \%$ of the cases, in contrast with older series in which the disease was commonly diagnosed after a year or more..$^{13}$ This difference probably reflects the current widespread use of CT scan in neurologic diagnosis. Although this method rarely shows pathognomonic images, ${ }^{,}$it often provides evidence for the diagnosis, ${ }^{5915^{-19}}$ as shown in figs 2 and 4 . As a result of the introduction of CT scanning, the number of cases of cysticercosis diagnosed in our department has doubled over the last 3 years.

The principal clinical manifestation in our series was intracranial hypertension. In $85 \%$ of these there was hydrocephalus due to cisternal or ventricular cysts or obstruction of subarachnoid spaces by diffuse basilar meningitis. This suggests that when intracranial hypertension is present, the main condition responsible is hydrocephalus, which carries in general a poor prognosis.

Seizures were observed in $40 \%$ of the cases, a figure similar to that reported in the literature on adult cysticercosis. ${ }^{291220} \mathrm{~A}$ recent report on childhood cysticercosis ${ }^{21}$ documented a higher incidence of seizures (75-82\%), probably reflecting the lower convulsive threshold in this age group in general. In the majority of our cases the seizures were generalised, and were commonly associated with intracranial hypertension or mental deterioration. Half of these patients underwent CT scanning, and parenchymatous cysts were found. The remaining patients were studied with plain skull radiographs and arteriography, and it is recognised that small cystic

Table 5 Surgical treatment in 27 patients with CNS cysticercosis

\begin{tabular}{llllrl}
\hline Type of Operation & \multicolumn{2}{l}{ Clinical result } & & Total \\
\cline { 2 - 5 } & Good & Poor & Dead & Unknown \\
\hline (1) Craniotomy with cyst resection & 6 & & & 1 & 7 \\
(2) Craniotomy with debridement of optic nerves plus shunt & 1 & & 1 & 2 \\
3) Posterior fossa craniotomy plus shunt & 4 & 1 & 6 & 5 & 16 \\
(4) CSF shunt alone & 11 & 1 & 8 & 7 & 27 \\
\hline
\end{tabular}


lasions can be missed by these procedures. The outcome was better in the seizure group, since only one of 11 such patients died.

Mental deterioration was observed as the initial manifestation in $22 \%$ of the cases. All these patients had hydrocephalus secondary to basilar meningitis. As previously reported, ${ }^{911}$ the parasite can also give rise to arteritis with secondary areas of necrosis of the parenchyma, probably another contributing factor to this clinical presentation.

In the past, conventional radiological examination (plain skull films, carotid angiography, pneumoencephalography) could detect only the rare large cysts or hydrocephalus. ${ }^{422}$ The more frequent multiple small intraparenchymatous cysts could not be diagnosed with these procedures. CT scan has become invaluable for the early and accurate detection of these lesions, ${ }^{81523}$ thus facilitating an early therapeutic decision. The CT aspects of cysticercosis have been amply delineated in several recent publications. ${ }^{915-19}$ These include: cystic lesions of low or high attenuation (non-calcified and calcified cysts, respectively), with or without concomitant hydrocephalus (fig $2 \mathrm{~A}$ ); low-attenuation cystic lesions with contrast ring enhancement after contrast injection (fig 2C); the pathognomonic image of cysts containing a calcified scolex (fig 4); demonstration of a "filling defect" in the cisternal or ventricular space with CT after metrizamide injection ${ }^{917}$ a procedure that has now replaced pneumoencephalography and ventriculography in the diagnosis of intraventricular or cisternal cysticercosis.

Prognosis of the disease is generally poor, a point well documented in the literature. ${ }^{3461220}$ The mortality rate in our series was $38 \%$. More than half of these patients died from basilar meningitis. By contrast, some forms of the disease, such as supratentorial parenchymatous cysts have a low mortality rate, and they have a relatively good prognosis if treated early (table 5).

We believe that craniotomy with cyst resection is indicated when there is a large solitary cyst or a group of cysts in a single focal area. Good results with this therapy have been reported. ${ }^{3}{ }^{1522}$ When dealing with multiple cysts in the 4 th ventricle or basal cisterns, craniotomy may be required in order to confirm the diagnosis. In addition, these lesions are known to adopt a basilar "racemose" form ${ }^{24}$ and are not likely to be cured by any therapy currently available. The rationale for their surgical resection is not only their tendency to produce obstructive hydrocephalus, but also the fact that death of the parasite is frequently followed by acute meningitis and ventriculitis, ${ }^{25}$ a complication that is often fatal.

We believe that in the presence of hydrocephalus caused by ventricular/cisternal cysts or by basilar cysticercosic meningitis-with or without supratentorial cysts - the CSF should be shunted as a form of palliative therapy. Although our results would indicate this is a method followed by poor results, it must be pointed out that only one death was due to shunt obstruction. In the remaining cases, death was caused by progression of the disease, rather than complications of therapy. The value of CSF shunting is cerebral cysticercosis has been stressed in two recent large series. ${ }^{911}$ It is expected that early detection of this condition by a high index of suspicion in populations at risk, along with the use of CT scanning, will improve the general outcome of patients afflicted by this disease.

\section{References}

' Brinck G. La Cisticercosis Cerebral. Estudio Anatomo Patologico y Clinico. Santiago (Chile): Leblanc, 1940.

${ }^{2}$ Arseni C, Samitca DC. Cysticercosis of the brain. $\mathrm{Br}$ Med J 1957;1:494-7.

${ }^{3}$ Asenjo A. Neurocysticercosis. Neurocirugia 1961;19:143-52.

${ }^{4}$ Cabieses F. Cysticercosis of the nervous system: III. Clinical findings and treatment. $J$ Neurosurg 1962;19:643-9.

${ }^{5}$ Latovitzki N, Abrams G, Clark C, Mayeux R, Ascherl G, Sciarra D. Cerebral cysticercosis. Neurology (Minneap.) 1978;28:838-42.

- Trelles JO, Trelles L. Cysticercosis of the Nervous System. In: Vinken PJ, Bruyn GW, eds. Handbook of Clinical Neurology, Vol 35. Amsterdam: NorthHolland Publ Co, 1978;291-320.

' Shanley JD, Jordan MC. Clinical aspects of CNS cysticercosis. Arch Int Med 1980;140:1309-13.

${ }^{8}$ Stern WE. Neurosurgical considerations of cysticercosis of the central nervous system. $J$ Neurosurg 1981;55:382-9.

${ }^{9}$ McCormick GF, Zee C-S, Heiden J. Cysticercosis cerebri. Review of 127 cases. Arch Neurol 1982;39:5349.

${ }^{10}$ Loo L, Braude A. Cerebral cysticercosis in San Diego. A report of 23 cases and a review of the literature. Medicine (Baltimore) 1982;61:341-59.

" Dixon H, Lipscomb F. Cysticercosis: an analysis and follow up of 450 cases. Med Res Council Spec Rep 1961;299:1-58.

${ }^{12}$ Stepien L. Cerebral cysticercosis in Poland: clinical symptoms and operative results in 132 cases. $J$ Neurosurg 1962;19:505-13.

${ }^{13}$ Schenone H, Ramirez R, Rojas A. Aspectos epidemiologicos de la neurocisticercosis en America Latina. Bol Chile Parasitol 1973;28:61-72.

${ }^{14}$ Keane JR. Neuro-ophthalmologic signs and symptoms of cysticercosis. Arch Ophthalmol 1982;100:1445-8.

${ }^{15}$ Carbajal JR, Palacios E, Azar-Kia B, Churchill R. Radiology of cysticercosis of the central nervous sys- 
tem including computed tomography. Radiology 1977;125: 127-31.

${ }^{16}$ Grisolia JS, Wiederholt WC. CNS cysticercosis. Arch Neurol 1982;39:540-4.

17 Madrazo I, Garcia Renteria JA, Paredes G, Olhagaray B. Diagnosis of intraventricular and cisternal cysticercosis by computerised tomography with positive intraventricular contrast medium. $J$ Neurosurg 1981;55:947-51.

${ }^{18}$ Byrd SE, Locke GE, Biggers S, Percy AK. The computed tomographic appearance of cerebral cysticercosis in adults and children. Radiology 1982;144:819-23.

${ }^{19}$ Minguetti G, Ferreira MVC. Computed tomography in neurocysticercosis. J Neurol Neurosurg Psychiat 1983;46:936-42.

${ }^{20}$ Pupo PP. Cysticercosis of the nervous system: clinical manifestations. Rev Neuropsiquiatr 1964;27:70-82.

${ }^{21}$ Lopez-Hernandez A, Garaizar C. Childhood cerebral cysticercosis: clinical features and computed tomographic findings in 89 Mexican children. Can J Neurol Sci 1982;9:401-7.

${ }^{22}$ Rowe FA, Youmans JR, Lee HJ, Cabieses F. Parasitic and Fungal Diseases of the Central Nervous System. In: Youmans JR, ed. Neurological Surgery, Vol 6, Philadelphia: WB Saunders, 1982;3373-6.

${ }^{23}$ Lobato RD, Lamas E, Portillo JM, et al. Hydrocephalus in cerebral cysticercosis. Pathogenic and therapeutic considerations. J Neurosurg 1981;55:786-93.

${ }^{24}$ Bickerstaff ER, Small JM, Woolf AL. Cysticercosis of the posterior fossa. Brain 1956;79:622-34.

${ }^{25}$ Madrazo I, Garcia Renteria JA, Sandoval M, Lopez Vega FJ. Intraventricular cysticercosis. Neurosurgery 1983;12:148-52. 